\title{
Ontology-Driven Conceptual Modeling
}

\author{
Christopher Welty \\ IBM Watson Research Center \\ Hawthorne, New York, USA \\ welty@us.ibm.com
}

\begin{abstract}
Ontology is a discipline of Philosophy concerned with what is, of the kinds and structures of objects, properties, events, processes and relations in every area of reality. The first recognition that the work of this philosophical field was relevant to designing and creating computer systems was made by John McCarthy in 1980, who claimed that one must first "list everything that exists - creating an ontology of our world." The computer science use of the term "ontology" has since undergone some evolution, and today it is normally taken as synonymous with knowledge engineering in AI, conceptual modeling in databases, and domain modeling in object-oriented design. This is not, however, simply a new word for something computer scientists have been doing for 20-30 years; Ontology is hundreds, if not thousands, of years old. We have been working for some time on adapting fundamental notions from this centuries-old field to the younger discipline of conceptual modeling, as the core of a formal (that is, domain independent) methodology for developing ontologies and evaluating ontological decisions. Among other things, we use our methodology to clean taxonomies by exposing inappropriate and inconsistent subumption relations.
\end{abstract}

